\title{
Bezlotoxumab for the prevention of Clostridium difficile infection: a review of current evidence and safety profile
}

This article was published in the following Dove Press journal: Infection and Drug Resistance

\author{
Carolyn D Alonso ${ }^{1,2}$ \\ Monica V Mahoney ${ }^{3}$ \\ 'Department of Medicine, Division \\ of Infectious Diseases, Beth Israel \\ Deaconess Medical Center, Boston, \\ MA, USA; ${ }^{2}$ Harvard Medical School, \\ Boston, MA, USA; ${ }^{3}$ Department of \\ Pharmacy, Beth Israel Deaconess \\ Medical Center, Boston, MA, USA
}

Correspondence: Carolyn D Alonso Department of Medicine, Division of Infectious Diseases, Beth Israel Deaconess Medical Center, II 0 Francis Street, Suite GB, Boston, MA 02215, USA Tel +l 6176327706

Fax +I 6176327626

Email calonso@bidmc.harvard.edu

\begin{abstract}
Clostridium difficile infection (CDI) is a leading nosocomial disease estimated to cause nearly half a million cases in the United States annually. Recurrent CDI (rCDI) affects $\sim 25 \%$ of patients after completion of standard of care therapy and is associated with substantial health care costs and a negative impact on patient's overall markers of quality of life. Bezlotoxumab is the first of its kind monoclonal antibody directed against $C$. difficile toxin B and indicated for prevention of rCDI in at-risk patients. For the present review, we assessed English-language studies evaluating the clinical efficacy, safety, and pharmacokinetics of bezlotoxumab in humans. Relevant studies were obtained through PubMed, Embase, Cochrane database library, Web of Science, and clinicaltrials.gov. Overall, bezlotoxumab demonstrated a $40 \%$ relative reduction rate (absolute rate reduction of $\sim 10 \%$ ) and a number needed to treat of 10 patients with a favorable safety profile. Special populations, including the elderly, immunocompromised, and patients with end-stage renal disease were evaluated in post hoc analyses with a similarly favorable reduction in rCDI. This review presents and interprets the most recent safety data and the clinical application of bezlotoxumab, highlighting specific high-risk patient populations.
\end{abstract}

Keywords: MK-6072, MDX-1388, Clostridium difficile monoclonal antibody, Clostridioides difficile, bezlotoxumab, Zinplava

\section{Introduction}

Clostridium difficile infection (CDI) is a major nosocomial bacterial disease with nearly half a million cases, 29,000 fatalities, and 83,000 first recurrences in the United States in 2011. ${ }^{1}$ The infection has been associated with a spectrum of clinical diseases ranging from mild, self-limited diarrhea to fulminant colitis and death in some cases. Increasing rates of infection have been noted in the United States over the last decade. ${ }^{2}$ This increase in rates has been associated with several highly virulent $C$. difficile strains, such as the BI/NAP1/027 strain, which demonstrate increased pathogenicity due to hypersecretion of toxins leading to colonic tissue damage. ${ }^{3}$ Following a primary episode of CDI, roughly $25 \%$ of patients will have a recurrent episode of infection (Table 1). ${ }^{4,5}$ After a first recurrence, rates of subsequent recurrence increase to $\sim 40 \%{ }^{6}$

Recurrent CDI has been associated with high financial costs. Dubberke et al ${ }^{7}$ evaluated nearly 4,000 hospitalized adult patients with CDI and determined that patients with recurrent $\mathrm{CDI}(\mathrm{rCDI})$ had higher attributable health care costs when compared to patients without recurrence in the 6 months following index CDI episode. On average, compared with patients without rCDI, patients with rCDI were estimated to accrue an 
Table I Proposed risk factors for recurrent Clostridium difficile infection (CDI)

\begin{tabular}{|c|c|}
\hline Host risk factors & $\begin{array}{l}\text { - Age } \geq 65 \text { years } \\
\text { - Low } C \text {. difficile-specific antitoxin antibody } \\
\text { levels } \\
\text { - Host genetics } \\
\text { - Compromised immune system } \\
\text { - Chronic renal failure } \\
\text { - Prior CDI episode }\end{array}$ \\
\hline $\begin{array}{l}\text { Severity of CDI } \\
\text { episode }\end{array}$ & - Severe primary CDI \\
\hline $\begin{array}{l}\text { Pathogen-specific } \\
\text { factors }\end{array}$ & $\begin{array}{l}\text { - Previous fluoroquinolone use } \\
\text { - Ongoing antibiotic use } \\
\text { - Proton pump inhibitors/antacids } \\
\text { - C. difficile strain type (ribotype } 027,078 \text {, } \\
\text { or } 244 \text { ) }\end{array}$ \\
\hline
\end{tabular}

Note: Data collated from References 2, 46, 47, 48, and 49.

additional \$11,631 in inpatient hospitalization costs. Zhang et $\mathrm{a}^{8}$ noted an average health care cost of $\$ 49,456$ and an attributable cost of $\$ 10,580$ in rCDI patients over the same follow-up period. Observational data from six U.K. acutecare hospitals also found substantial costs associated with rCDI when compared to a single episode of CDI, ${ }^{9}$ primarily driven by hospital length of stay. Overall markers of quality of life including mobility, pain and discomfort, and anxiety and depression were also worse in the CDI population compared with age-matched population norms as assessed by a prospective patient self-assessment tool.

The pathogenesis of $C$. difficile infection involves several sequential steps. In most cases, antibiotic exposure alters the intestinal microbiota leading to microbial dysbiosis with subsequent exposure and then colonization by the organism. Once the organism has colonized the colonic tract, two major toxins known as the "enterotoxin" or toxin A and the "cytotoxin" or toxin B are transcribed and elaborated by the organism. A third toxin, known as the "binary toxin," is also produced, although its function is less well understood. Toxins A and B belong to a class of Clostridial toxins that cause colonic tissue damage to the enteric cytoskeletal wall and disruption of the tight junctions that connect colonic cells..$^{10}$ Changes to the cell wall combined with secretion of inflammatory mediators result in recruitment of neutrophils to the site of infection. Therefore, toxin is thought to play a critical role in clinical disease.

It was noted in the early 2000 s that the host immune response to the pathogen, specifically production of serum antitoxin immunoglobulin, was likely necessary for patients in whom asymptomatic carriage occurred. ${ }^{11}$ Subsequently, antibody response to $C$. difficile toxin was also noted to be necessary for prevention of rCDI. ${ }^{12}$ In a leading model proposed by Zhang et al, ${ }^{13}$ systemic antitoxin antibodies are hypothesized to protect against $C$. difficile toxins by direct toxin neutralization in the colonic lumen. In the noninflamed, healthy intestinal tract, antibodies concentrate on the basolateral gut epithelium, but are thought to have limited leakage into the gut lumen. However, in the presence of $C$. difficile toxins, damage to luminal epithelial cells occurs, leading to changes in the cellular cytoskeleton and passage of antitoxin antibodies into the gut lumen. There they are able to directly neutralize toxin, prevent further tissue damage, and allow for restoration of the epithelial layer.

This review focuses on the current evidence and safety profile behind the novel anti-C. difficile toxin B monoclonal antibody bezlotoxumab, with a focus on its place in therapy for prevention of rCDI. The current review presents safety data and the clinical application of the drug, highlighting specific high-risk patient populations.

\section{Materials and methods}

A search of PubMed (1946 to August 2018) using the search terms "bezlotoxumab", "Zinplava", "MK-6072”, "MDX1388", "Clostridium difficile or Clostridioides difficile and monoclonal antibody", and "Clostridium difficile or Clostridioides difficile and antitoxin" was performed. All data available in English were reviewed, focusing on treatment and safety data in humans and animals. We performed additional reviews using Embase, Cochrane database library, Web of Science, clinicaltrials.gov, and abstracts from major infectious diseases and gastroenterology conference proceedings.

\section{Clinical trials involving bezlotoxumab}

To date, there have been five Phase I studies, two Phase II trials, and two Phase III trials, which have investigated bezlotoxumab. ${ }^{14}$ Early product development also included antitoxin A antibody (actoxumab) as it was hypothesized at the time of investigational drug application in 2005 that either antitoxin A antibody alone or the combination of antitoxin A antibody plus antitoxin B antibody might be most efficacious in the prevention of recurrence. Subsequently, the actoxumab program was discontinued following an interim analysis of MODIFY I (see "Efficacy" section below).

Bezlotoxumab is a fully humanized monoclonal antibody that only binds to $C$. difficile toxin B. Binding to toxin B neutralizes the toxin and prevents damage to mammalian colonic cells. ${ }^{15,16}$ The Food and Drug Administration (FDA)-approved dose is a single $10 \mathrm{mg} / \mathrm{kg}$ dose administered intravenously over 60 minutes during active 
anti-C. difficile therapy, up to treatment day 14. Bezlotoxumab is eliminated by catabolism and therefore does not require dosage adjustment in either renal or hepatic impairment. As drug clearance increases with increasing body weight, dosing on actual body weight is recommended. Plasma half-life is reported as 19 days following a single dose. ${ }^{16}$ During Phase II and III studies, no antibezlotoxumab antibodies were detected in patients receiving the medication. ${ }^{14,17,18}$ There are no carcinogenicity, genotoxicity, or fertility studies published. No drug-drug or druglaboratory interactions are anticipated or published, due to the catabolic elimination. ${ }^{15}$

\section{Efficacy}

MODIFY I and II were Phase III, double-blind trials comparing bezlotoxumab to placebo as add-on therapy to 10-14 days of standard of care (SOC) for CDI. Eligibility criteria included age $\geq 18$ years and a diagnosis of CDI (diarrhea plus positive toxigenic $C$. difficile test). Patients were randomized to single-dose bezlotoxumab $10 \mathrm{mg} / \mathrm{kg}$, actoxumab $10 \mathrm{mg} / \mathrm{kg}$ (MODIFY I only), actoxumab/bezlotoxumab $10 \mathrm{mg} / \mathrm{kg}$ each, or placebo. Doses were administered any time from day 0 to day 14 of active anti-CDI therapy. SOC was at the discretion of the treating physician and included metronidazole, vancomycin ( \pm intravenous metronidazole), or fidaxomicin ( \pm intravenous metronidazole). The primary endpoint was rCDI up to 12 weeks after initial cure in the modified intention-to-treat (mITT) population. Secondary analyses included global cure and prespecified subgroup analyses such as older patients, immunocompromised, severe CDI, and previous history of CDI. Global cure was defined as cure of baseline CDI with no recurrence. ${ }^{17}$

Overall, 1,613 patients were randomized with 1,554 patients included in the mITT population. CDI recurred in $16.5 \%$ of bezlotoxumab vs $26.6 \%$ of placebo patients in the combined MODIFY I and II studies, representing a $40 \%$ relative reduction rate $(P<0.0001)$ and a number needed to treat of 10 patients. Similar results were reported in the individual trials. Bezlotoxumab had no effect on clinical cure (clinical cure of $80 \%$ bezlotoxumab vs $80 \%$ placebo). Of note, an interim analysis of MODIFY I indicated a lack of efficacy and increased mortality due to sepsis in the actoxumab arm; therefore, this arm was terminated early, and results were primarily focused on the bezlotoxumab compared to placebo arms. Global cure was higher in bezlotoxumab vs placebo patients $(63.5 \%$ vs $53.7 \%$, respectively, $P=0.0001)$. This finding was mostly driven by the MODIFY II subset. In the prespecified subgroups, bezlotoxumab had lower recur- rence rates than placebo in all but the more virulent strains (ribotypes 027, 078, or 244). ${ }^{17}$

Adverse drug reactions (ADRs) were mild or moderate and comparable between groups. Infusion-related reactions were most common, in $10.3 \%$ and $7.6 \%$ of bezlotoxumab and placebo patients. Approximately $61 \%$ of patients reported at least one ADR within the 4 weeks following the infusion, including nausea, diarrhea, pyrexia, and headache. ${ }^{17}$

\section{Medication administration}

For medication administration information, please refer to Table 2.

\section{Safety}

Bezlotoxumab is generally well tolerated with infusionrelated reactions $(10 \%)$ - the most commonly reported ADRs. ADRs within 4 weeks of administration reported in $\geq 4 \%$ of patients in the MODIFY I and II trials included nausea (7\%), pyrexia (5\%), and headache (4\%). These did not differ significantly from placebo. ${ }^{15}$

Serious ADRs were reported in $29 \%$ of bezlotoxumab and $33 \%$ of placebo patients within 12 weeks of infusion. The

Table 2 Bezlotoxumab characteristics ${ }^{15,19,50}$

\begin{tabular}{|l|l|}
\hline Pharmacokinetics parameter & Result \\
\hline Clearance $(\mathrm{Cl})$ & $0.3 \mathrm{I} 7 \mathrm{~L} / \mathrm{day}$ \\
Mean volume of distribution $(\mathrm{Vd})$ & $7.33 \mathrm{~L}$ \\
Elimination half-life $\left(t_{1 / 2}\right)$ & 19 days \\
AUC $_{0-\infty}$ & $53,000 \mathrm{mcg} \times$ hours $/ \mathrm{mL}$ \\
$C_{\max }$ & $185 \mathrm{mcg} / \mathrm{mL}$ \\
Dosage and administration & $10 \mathrm{mg} / \mathrm{kg}$ \\
$\quad$ Dose & During active CDI treatment; \\
Timing & usually days $0-14$ \\
& Intravenous \\
Route & 1 hour \\
Infusion time & Use $0.2-5 \mu \mathrm{m}$ in-line or add- \\
Special instructions & on filter \\
Supplied & \\
I Vial ( $50 \mathrm{~mL})$ & $1,000 \mathrm{mg}$ bezlotoxumab in 40 \\
& $\mathrm{~mL}$ solution \\
Preparation & \\
Compatible solutions & $0.9 \%$ sodium chloride of \\
Final concentration & dextrose $5 \%$ water \\
Storage & $\mathrm{I}-10 \mathrm{mg} / \mathrm{mL}$ \\
Undiluted & \\
Diluted, room temperature & Refrigerated \\
Diluted, refrigerated $\left(2^{\circ} \mathrm{C}-8^{\circ} \mathrm{C} ;\right.$ & 16 hours \\
$\left.36^{\circ} \mathrm{F}-46^{\circ} \mathrm{F}\right)$ & 24 hours \\
Cost (AWP) & \\
I Vial $(\mathrm{I}, 000 \mathrm{mg})$ & $\$ 4,500$ \\
\hline
\end{tabular}

Abbreviations: $\mathrm{AUC}_{0-\infty}$, area under the concentration-time curve from time zero to infinity; AWP, average wholesale price; $C D I$, Clostridium difficile infection; $C_{\max }$ maximum drug concentration. 
most common serious ADRs were sepsis, pneumonia, acute kidney injury, urinary tract infections, and heart failure. ${ }^{15,19}$ Mortality rates within 12 weeks of infusion were $7.1 \%$ in bezlotoxumab- and $7.6 \%$ in placebo-treated patients.

Heart failure was not seen in preclinical trials but reported in $17(2.2 \%)$ bezlotoxumab and $7(0.9 \%)$ placebo treated patients in Phase III trials. Heart failure was more frequently observed in patients with a history of congestive heart failure (CHF). Additionally, patients with CHF were more likely to report increased treatment-emergent ADRs (83.9\% vs $70.2 \%$ ), serious ADRs (53.4\% vs $48 \%$ ), and deaths ( $19.5 \%$ vs $12.5 \%$ ) than placebo-treated patients. Death from cardiovascular diseases was also higher in bezlotoxumabtreated patients $(8[14.3 \%]$ vs $4[6.8 \%]){ }^{14,17,19}$ Due to these findings, the use of bezlotoxumab is cautioned in patients with $\mathrm{CHF}$, with careful weight of risks of ADRs vs benefits of preventing rCDI. ${ }^{15}$

\section{Efficacy relative to administration timing}

A post hoc analysis of the MODIFY I and II trials determined that there was no difference in rCDI if bezlotoxumab was administered on days $0-2,3-4$, or $\geq 5$ days after the initiation of SOC (rCDI rates 19.3\%, 20.4\%, and 22.8\%, respectively). ${ }^{20}$ Additionally, earlier administration of bezlotoxumab did not impact the time-to-diarrhea resolution. These results lend support to deferring administration of bezlotoxumab to the outpatient setting, potentially improving third-party reimbursement, as long as treatment for CDI is still active.

\section{Thirty-day readmission rates}

Hospital readmissions due to CDI can be taxing on the health care system. Patients with rCDI are 12.5 times more likely to incur inpatient costs compared to patients without recurrences. ${ }^{7}$ Therefore, decreasing readmission rates may help reduce costs associated with rCDI.

Bezlotoxumab has been shown to decrease 30-day hospital readmission rates by $6 \%$ compared to placebo, when added to SOC. Data from 530 bezlotoxumab and 520 placebo mITT patients who were hospitalized at time of enrollment in the MODIFY I and II trials were used to estimate readmission rates. All-cause 30-day readmission was defined as any admission within 30 days of discharge. CDI-associated 30-day readmission was defined as readmission within 5 days after a new episode of CDI, new CDI onset during readmission, or the discharge diagnosis included terms synonymous with CDI, rCDI, or pseudomembranous colitis as recorded on the trial case report form. Subgroup analyses were performed on patients at high risk for relapse, including older age, severe
CDI, rCDI, infection due to the 027 strain, and immunocompromise. All-cause readmission were lower for bezlotoxumabassociated (absolute difference, $-3.7 \%$; 95\% CI, -9.0 to 1.5 , relative difference, $-12.1 \%$ ) as well as CDI-associated readmissions (absolute difference, $-6.1 \%$; 95\% CI, -9.5 to -2.8 ; relative difference, $-53.4 \%)$. ${ }^{21}$ In evaluating high-risk subgroups, only older age and severe CDI demonstrated statistically lower readmission rates for bezlotoxumab patients.

\section{Special populations}

After publication of the initial MODIFY clinical trials, several subsequent reports have been presented and published that have further evaluated rCDI high-risk patient populations. These data provide a foundation for identification of the patient populations most likely to benefit from this novel agent. The reduction in $\mathrm{rCDI}$ in special populations treated with bezlotoxumab is presented in Figure 1.

\section{Identifying the highest risk rCDI patient population}

A 2018 post hoc analysis evaluated the efficacy of bezlotoxumab in patients with previously identified "high-risk" rCDI risk factors including age $\geq 65$ years, compromised immunity, severe CDI, prior CDI episode(s), and infection with ribotypes $027 / 078 / 244 .{ }^{22}$ Overall, there were 1,554 patients included in the mITT. Clinical outcomes included rCDI in 12 weeks, fecal microbiota transplant procedures (FMT), 30-day all-cause and CDI-associated hospital readmissions, and mortality at 30 and 90 days after randomization. Treatment with bezlotoxumab did not affect initial clinical cure rates in patients receiving SOC antibiotics for CDI. Patients treated with bezlotoxumab experienced the following reductions in CDI recurrence for each of the risk factor categories: $-20.1 \%$ (range $-27.0,-13.2$ ) for age $\geq 65$ years, $-17.9 \%$ (range $-27.7,-7.6$ ) for history of CDI, $-17.0 \%(-28.0,-6.0)$ for immunocompromised host, $-15.6 \%$ (range $-28.0,-2.8$ ) for severe CDI, and $-12.9 \%$ (range -26.8, 1.6) for infection with ribotypes 027/078/244. All of the categories demonstrated a statistically significant reduction in CDI recurrence, with the exception of infection with ribotypes $027 / 078 / 244$. When further stratified by number of underlying risk factors, there was a greater impact on prevention of CDI recurrence as the number of risk factors increased. Patients with more than or equal to three risk factors experienced the greatest reduction in CDI recurrence with bezlotoxumab ( $-24.8 \%$ [range $-39.1 \%,-9.3 \%]$ ), suggesting a potential targeted patient population for the drug. One limitation of these data is that several of the risk factors are loosely defined, including immunocompromised patients, which was based on clinical criteria. 


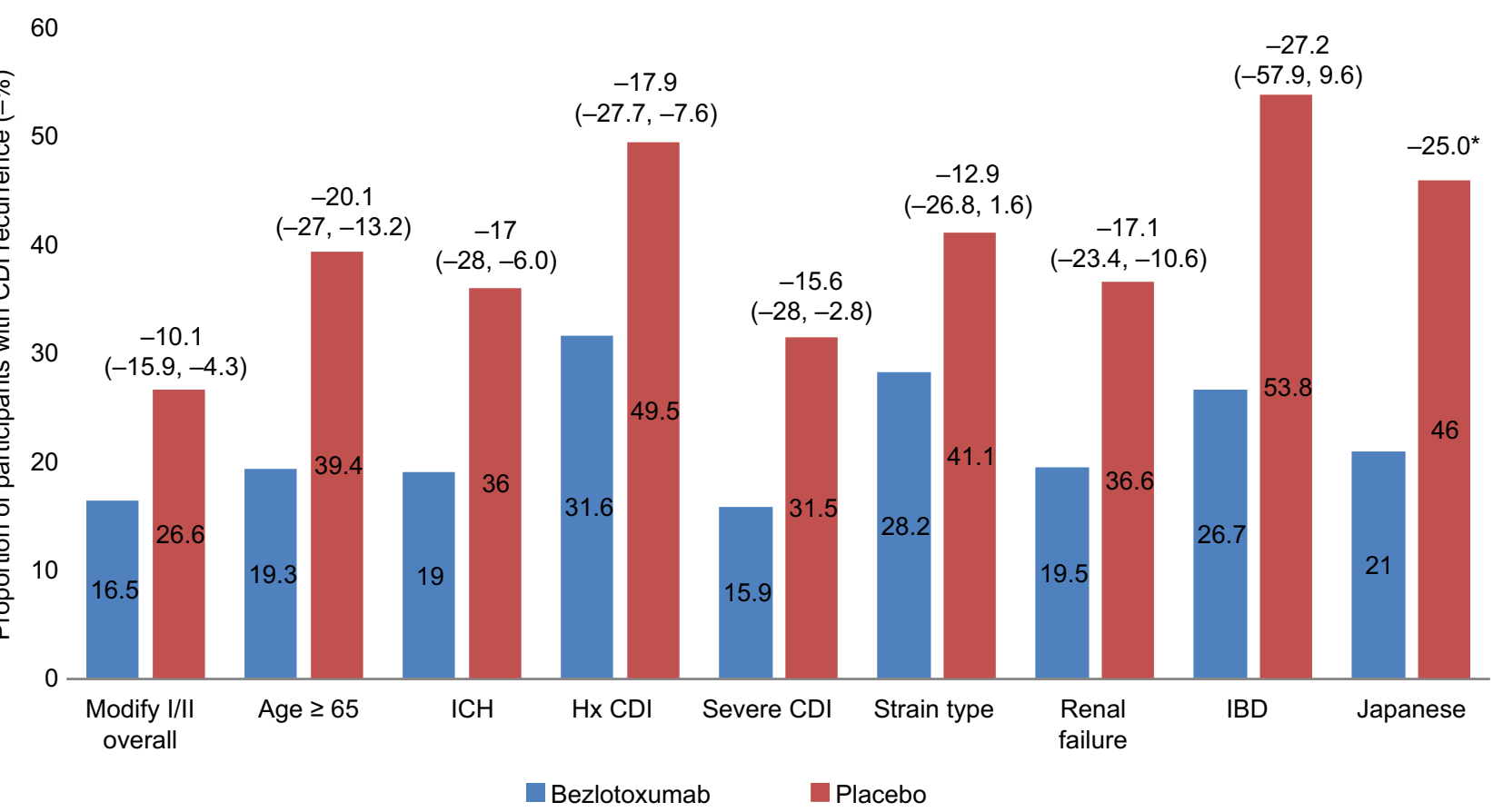

Figure I Absolute reduction in recurrent $\mathrm{CDI}(\mathrm{rCDI})$ in special populations treated with bezlotoxumab.

Notes: CDI recurrence rates are noted within the colored bars for each population of interest. Overall reduction in recurrence rate is noted above the bars with $95 \% \mathrm{Cl}$ noted in parentheses. *Data from the Japanese study did not provide $95 \% \mathrm{Cl}$. The $P$-value for the difference between bezlotoxumab-treated group and placebo group was $0.0197 \cdot 18,22,29,33,35$

Abbreviations: CDI, C. difficile infection; ICH, immunocompromised host; Hx CDI, history of CDI; IBD, inflammatory bowel disease.

\section{Patients receiving concomitant antibiotics}

In addition to the rCDI risk factors evaluated in the above study, data presented at the 2016 IDWeek conference evaluated the efficacy of bezlotoxumab in prevention of CDI recurrence in patients receiving concomitant antibiotics. ${ }^{23}$ Prior data have demonstrated that concurrent non-CDI antibiotics given during SOC treatment have been associated with CDI treatment failure and risk of rCDI, likely due to continued interruption of the normal bacterial flora. ${ }^{24,25}$ Pooled clinical data from the MODIFY I and II studies demonstrated frequent use of concomitant antibiotics (37\% in the bezlotoxumab arm vs $41 \%$ in the placebo arm) within the follow-up period (defined as the first day after the end of SOC treatment through onset of rCDI or day 90 following the infusion of study medication). rCDI was observed in $18 \%$ of bezlotoxumab-treated patients who received concomitant antibiotics compared with $28 \%$ of placebo subjects receiving concomitant antibiotics. These preliminary data suggest that the efficacy of bezlotoxumab was maintained in patients receiving concomitant antibiotics.

\section{Patients with renal failure}

Renal failure, particularly stage 3 chronic kidney disease (CKD) and higher, has been associated with worse clinical outcomes in CDI including more difficult to treat infection as well as increased risk for CDI recurrence. ${ }^{26,27}$ In a post hoc analysis of two randomized clinical trials that compared fidaxomicin to vancomycin for treatment of CDI, investigators evaluated CDI recurrence within 28 days of completion of fidaxomicin or vancomycin. In this work, they found that stage 3 or greater CKD conferred a statistically significant increased risk for CDI recurrence, even after controlling for factors linked to recurrence such as age, type of antibiotic treatment, concomitant antibiotics, and laboratory parameters such as white blood cell count. This elevated CDI risk may be related to frequent health care contacts, antibiotic exposures, or other host immune defects in the setting of uremia. Endstage renal disease has also been associated with higher per patient costs for CDI episodes and higher patient mortality, ${ }^{28}$ making this patient population a targeted one for preventative therapies such as bezlotoxumab.

Preliminary data presented at IDWeek 2017 investigated CDI outcomes in bezlotoxumab-treated patients with significant renal impairment. ${ }^{29}$ These data were derived from a post hoc analysis of the MODIFY I and II studies. Of the 1,554 patients included in the study, 430 patients had substantial renal impairment (defined as a glomerular filtration rate $<60$ $\mathrm{mL} / \mathrm{min} / 1.73 \mathrm{~m}^{2}$ ). These patients tended to be older, were more likely to be immunocompromised, and received more 
concomitant antibiotics. In this high-risk patient population, bezlotoxumab was associated with a -17.1 percentage point decrease in rCDI (range, -23.4, -10.6) compared with patients who received placebo (rCDI rate in bezlotoxumabtreated group $19.5 \%$ vs $36.6 \%$ in the placebo group). Further published data is expected (Y Golan, personal communication, July, 2018).

\section{Inflammatory bowel disease (IBD)}

Historically, IBD has been linked to higher CDI rates and more difficult to treat infection. ${ }^{30-32}$ However, patients with these underlying conditions have been frequently excluded from CDI clinical trials due to underlying immunosuppression. The MODIFY I and II trials included 44 participants in the mITT population with IBD (23 with ulcerative colitis, 18 with Crohn's disease, and 3 with noncharacterized IBD). In a recently presented post hoc analysis of these data, investigators found differences in the baseline characteristics between IBD patients and non-IBD patients in the MODIFY studies. ${ }^{33,34}$ The IBD patients were more likely to be younger, immunocompromised, diagnosed as an outpatient, have less recent antibiotic exposure, and were more likely to experience rCDI compared with the non-IBD population. There was a $27.2 \%$ absolute reduction $\mathrm{rCDI}$ in bezlotoxumab-treated IBD patients compared with patients who received placebo. However, the $95 \%$ CIs $(-57.9,9.6)$ overlapped between groups. The study was limited by small sample size. Further larger-scale studies will be needed to determine if there is a benefit of bezlotoxumab for rCDI in the IBD population.

\section{Effect in Japanese patients}

Recent post hoc analysis of the MODIFY II study investigated the role of bezlotoxumab in the 95 Japanese patients included in the trial. ${ }^{35}$ Overall, Japanese patients tended to be older (91\% Japanese patients were $\geq 65$ years of age compared with $53 \%$ in the overall study population), with a higher percentage of severe CDI (24\% in the Japanese group compared with $16 \%$ in the overall population), and were more likely to be inpatients at the time of CDI diagnosis. Rates of rCDI were also significantly higher in the Japanese placebo cohort (Japanese: $46 \%$, overall: $27 \%$ ). Strain type also differed compared with global data, with no hypervirulent strains (ribotypes 027, 078, or 244) identified. In the Japanese population, the overall efficacy of bezlotoxumab was favorable (rCDI rate of $21 \%$ bezlotoxumab vs $46 \%$ placebo, $P=0.0393$ ) compared with the larger MODIFY I and II studies (rCDI rate $16.5 \%$ in bezlotoxumab arm and $26.6 \%$ placebo arm). Despite there being higher baseline recurrence rates in the Japanese placebo and bezlotoxumab-treated patients compared with the larger MODIFY II patient population, the authors concluded that bezlotoxumab may have a beneficial effect in reducing rCDI in an older Japanese CDI population.

\section{Pediatrics}

As the MODIFY I and II studies excluded individuals younger than 18 years, the safety and efficacy of bezlotoxumab in pediatric populations have not been evaluated. ${ }^{15}$ MODIFY III is a global randomized, double-blind, placebo-controlled clinical trial to evaluate the safety, tolerability, pharmacokinetics, and efficacy of bezlotoxumab in children ages 1 to $<18$ years. ${ }^{36}$ The study is presently recruiting subjects with an estimated completion date of May 2022.

\section{Response to bezlotoxumab may relate to host genetics}

Several small studies have suggested a possible link between susceptibility to CDI and host genetics. ${ }^{37-39}$ Host genetics may also influence risk of recurrence. ${ }^{40}$ MODIFY I and II participants who provided consent for genetic testing and who achieved initial clinical cure were evaluated in an exploratory pharmacogenetic analysis to determine genetic markers associated with improved response to bezlotoxumab. ${ }^{41}$ The genome-wide association study found a statistically significant reduction in rCDI in those bezlotoxumab-treated participants who carried the $\mathrm{T}$ allele of the single nucleotide polymorphism, rs2516513. While preliminary, these data suggest a future genetic marker to identify patients with the greatest likelihood of bezlotoxumab treatment response. Additionally, this may identify patients who are unlikely to benefit from the drug and in whom alternative therapies for recurrence prevention should be utilized.

\section{Cost effectiveness}

All-cause cost associated with CDI hospital stays is estimated at $\$ 8.2$ billion. $^{42,43}$ Each additional hospitalization beyond the initial CDI course is estimated to cost an extra $\$ 11,146 .^{7}$ While proven to decrease rCDI, the acquisition cost of bezlotoxumab, at $\sim \$ 4,500 / 1,000 \mathrm{mg}$ vial, limits its utilization. Two cost-effective analyses of bezlotoxumab were recently completed, focusing on slightly different patient populations and treatment options.

The first analysis used MODIFY I and II data from subgroups at high risk for relapse, including older age, immunocompromised patients, and severe CDI subgroups. These groups were further divided into first occurrence and 
any recurrence. The model predicted that bezlotoxumab can decrease first recurrence by $10.1 \%$, total recurrences by $16.7 \%$, and 180 -day mortality by $1.1 \%$ compared to SOC. At a willingness-to-pay threshold of $\$ 50,000 /$ quality-adjusted life-years (QALY) gained, there was a $96.7 \%$ probability that the addition of bezlotoxumab would be cost effective. ${ }^{21}$ Per patient discounted incremental costs associated with bezlotoxumab use were $\$ 2,444$; total number of QALYs increased by 0.12 per patient, with an incremental cost-effectiveness ratio (ICER) of $\$ 19,824$ / QALY gained. In the subgroup analyses, the ICERs were $\$ 15,298 /$ QALY for age $\geq 65$ years, $\$ 12,597 /$ QALY for immunocompromised, and \$21,430/QALY for severe CDI. The ICERs for patients with rCDI were not as robust, with only $\$ 3,591 /$ QALY for age $\geq 65$ years, $\$ 4,979 /$ QALY for immunocompromised, and $\$ 2,938$ for severe CDI. ${ }^{44}$

The second analysis focused on patients with rCDI only (first recurrence) and evaluated three potential treatment options: oral vancomycin alone, oral vancomycin plus bezlotoxumab, or fidaxomicin alone. Additional considerations for vancomycin tapers and FMT were factored in for second and beyond recurrences, modeling a more real-world treatment approach. At a willingness-to-pay threshold of $\$ 100,000 /$ QALY gained, there was a $68.4 \%$ probability that vancomycin alone would be the most cost effective, followed by a $29.2 \%$ probability of fidaxomicin and only $2.4 \%$ probability of vancomycin plus bezlotoxumab. The model predicted that vancomycin alone was associated with a QALY gained of 0.8019. Fidaxomicin was associated with a QALY gained of 0.8046 and an ICER of $\$ 500,975$. The bezlotoxumab regimen was associated with a higher cost than fidaxomicin and lower QALY gained, therefore, deemed not likely to be cost effective. ${ }^{45}$ Therefore, the authors concluded that vancomycin alone was the most cost effective at a threshold of $\$ 100,000 /$ QALY gained.

\section{Place in therapy, future studies, and unanswered questions}

In October 2016, the FDA approved bezlotoxumab for prevention of CDI recurrence in adult patients at risk of rCDI. The drug became commercially available for clinical use in February 2017. Due to the recent product availability of this drug, it has not yet been incorporated into any of the major CDI guidelines.

\section{Unanswered questions}

Many unanswered questions remain regarding bezlotoxumab's place in therapy: How will bezlotoxumab compare head to head with FMT, fidaxomicin, or other novel CDI therapies in high-risk rCDI populations? Will there be any difference in safety signals between bezlotoxumab and the treatment modalities such as FMT? Using real-world clinical data, how will bezlotoxumab compare to FMT in terms of cost to the patient and the health care system? Are there certain genetic or host factors that may predict who may benefit the most from this therapy? How will the drug perform in specialized populations such as hematologic malignancy patients, bone marrow transplant patients, and solid organ transplant recipients? Will the drug have a role in prevention of CDI in pediatric populations?

One of the notable weaknesses is that much of the published primary data come from the drug pharmaceuticalsponsored MODIFY trials. The majority of the data for special populations come from post hoc analyses, which have been presented in poster form at scientific meetings. These data are yet to be published in peer-reviewed journals. Realworld clinical experience and independent investigations will be helpful to verify the clinical efficacy in high-risk populations.

\section{Conclusion}

Bezlotoxumab is the first of its kind, fully humanized monoclonal antibody directed against $C$. difficile toxin B and indicated for prevention of $\mathrm{rCDI}$ in at-risk patients. Its novel mechanism of action, apparent lack of impact on the fecal microbiome, and safety profile make it an attractive adjunctive therapy for prevention of rCDI. Over the next several years, real-world clinical data should shed light on its efficacy in the highest risk CDI populations and may provide further insight into how the drug compares with other novel preventative therapies.

\section{Author contributions}

Both authors contributed to data analysis, drafting and revising the article, gave final approval of the version to be published, and agree to be accountable for all aspects of the work.

\section{Disclosure}

Dr Alonso has acted as a scientific advisor to Merck and Roche. Dr Alonso has received research funding from Merck. Dr Alonso has NIH Loan Repayment Funding through the National Institute of Allergy and Infectious Diseases (NIAID). Dr Mahoney has served as a consultant for Roche Diagnostics, Tetraphase Pharmaceuticals Inc., Melinta Therapeutics, and CutisPharma. Dr Mahoney has also received 
research funding from Durata Therapeutics and Merck. The authors report no other conflicts of interest in this work.

\section{References}

1. Lessa FC, Mu Y, Bamberg WM, et al. Burden of Clostridium difficile infection in the United States. N Engl J Med. 2015;372(9):825-834.

2. Centers for Disease Control and Prevention [webpage on the Internet]. Clostridium difficile infection (CDI) tracking. 2017. Available from: https://www.cdc.gov/hai/eip/clostridium-difficile.html. Accessed August 3, 2018.

3. Kociolek LK, Gerding DN. Clinical utility of laboratory detection of Clostridium difficile strain BI/NAP1/027. J Clin Microbiol. 2016;54(1):19-24.

4. Johnson S, Louie TJ, Gerding DN, et al. Vancomycin, metronidazole, or tolevamer for Clostridium difficile infection: results from two multinational, randomized, controlled trials. Clin Infect Dis. 2014;59(3):345-354.

5. Louie TJ, Miller MA, Mullane KM, et al. Fidaxomicin versus vancomycin for Clostridium difficile infection. $N$ Engl $J$ Med. 2011;364(5):422-431.

6. Sheitoyan-Pesant C, Abou Chakra CN, Pépin J, Marcil-Héguy A, Nault $\mathrm{V}$, Valiquette L. Clinical and healthcare burden of multiple recurrences of Clostridium difficile infection. Clin Infect Dis. 2016;62(5):574-580.

7. Dubberke ER, Schaefer E, Reske KA, Zilberberg M, Hollenbeak CS, Olsen MA. Attributable inpatient costs of recurrent Clostridium difficile infections. Infect Control Hosp Epidemiol. 2014;35(11):1400-1407.

8. Zhang D, Prabhu VS, Marcella SW. Attributable healthcare resource utilization and costs for patients with primary and recurrent Clostridium difficile infection in the United States. Clin Infect Dis. 2018;66(9):1326-1332.

9. Wilcox MH, Ahir H, Coia JE, et al. Impact of recurrent Clostridium difficile infection: hospitalization and patient quality of life. JAntimicrob Chemother. 2017;72(9):2647-2656.

10. Rupnik M, Wilcox MH, Gerding DN. Clostridium difficile infection: new developments in epidemiology and pathogenesis. Nat Rev Microbiol. 2009;7(7):526-536.

11. Kyne L, Warny M, Qamar A, Kelly CP. Asymptomatic carriage of Clostridium difficile and serum levels of IgG antibody against toxin A. N Engl J Med. 2000;342(6):390-397.

12. Kyne L, Warny M, Qamar A, Kelly CP. Association between antibody response to toxin $\mathrm{A}$ and protection against recurrent Clostridium difficile diarrhoea. Lancet. 2001;357(9251):189-193.

13. Zhang Z, Chen X, Hernandez LD, et al. Toxin-mediated paracellular transport of antitoxin antibodies facilitates protection against Clostridium difficile infection. Infect Immun. 2015;83(1):405-416.

14. Federal Drug Administration [webpage on the Internet]. FDA briefing document bezlotoxumab injection meeting of the Antimicrobial Drugs Advisory Committee (AMDAC); 2016. Available from: http://www. fda.gov/downloads/AdvisoryCommittees/CommitteesMeetingMaterials/Drugs/AntiInfectiveDrugsAdvisoryCommittee/UCM505290.pdf. Accessed December 10, 2018.

15. Merck Sharp \& Dohme Corp [webpage on the Internet]. Whitehouse Station, New Jersey. Zinplava (bezlotoxumab) Prescribing Information; 2016. Available from: www.accessdata.fda.gov/drugsatfda_docs/ label/2016/761046s000lbl.pdf. Accessed August 9, 2018.

16. Yang Z, Ramsey J, Hamza T, et al. Mechanisms of protection against Clostridium difficile infection by the monoclonal antitoxin antibodies actoxumab and bezlotoxumab. Infect Immun. 2015;83(2):822-831.

17. Wilcox MH, Gerding DN, Poxton IR, et al. Bezlotoxumab for prevention of recurrent Clostridium difficile infection. $N$ Engl J Med. 2017;376(4):305-317.

18. Lowy I, Molrine DC, Leav BA, et al. Treatment with monoclonal antibodies against Clostridium difficile toxins. $N$ Engl $J$ Med. 2010;362(3):197-205.

19. Chapin RW, Lee T, McCoy C, Alonso CD, Mahoney MV. Bezlotoxumab: could this be the answer for Clostridium difficile recurrence? Ann Pharmacother. 2017;51(9):804-810.
20. Birch T, Golan Y, Rizzardini G, et al. Efficacy of bezlotoxumab based on timing of administration relative to start of antibacterial therapy for Clostridium difficile infection. J Antimicrob Chemother. 2018;73(9):2524-2528.

21. Prabhu VS, Dubberke ER, Dorr MB, et al. Cost-effectiveness of bezlotoxumab compared with placebo for the prevention of recurrent Clostridium difficile infection. Clin Infect Dis. 2018;66(3):355-362.

22. Gerding DN, Kelly CP, Rahav G, et al. Bezlotoxumab for prevention of recurrent Clostridium difficile infection in patients at increased risk for recurrence. Clin Infect Dis. 2018;67(5):649-656.

23. Mullane K, Wilcox M, Golan Y, et al. Efficacy of bezlotoxumab (bezlo) in prevention of Clostridium difficile infection recurrence ( $\mathrm{rCDI}$ ) in patients receiving concomitant systemic antibiotics (CAs). Open Forum Infect Dis. 2016;3(Suppl 1):2115.

24. Nair S, Yadav D, Corpuz M, Pitchumoni CS. Clostridium difficile colitis: factors influencing treatment failure and relapse - a prospective evaluation. Am J Gastroenterol. 1998;93(10):1873-1876.

25. Mullane KM, Miller MA, Weiss K, et al. Efficacy of fidaxomicin versus vancomycin as therapy for Clostridium difficile infection in individuals taking concomitant antibiotics for other concurrent infections. Clin Infect Dis. 2011;53(5):440-447.

26. Bárány P, Stenvinkel P, Nord CE, Bergström J. Clostridium difficile infection - a poor prognostic sign in uremic patients? Clin Nephrol. 1992;38(1):53-57.

27. Mullane KM, Cornely OA, Crook DW, et al. Renal impairment and clinical outcomes of Clostridium difficile infection in two randomized trials. Am J Nephrol. 2013;38(1):1-11.

28. Goyal A, Chatterjee K, Yadlapati S, Rangaswami J. Impact of end stage kidney disease on costs and outcomes of Clostridium difficile infection. Int J Infect Dis. 2017;62:8-9.

29. Golan Y, Dupont HL, Aldomiro F, Jensen EH, Hanson ME, Dorr MB. Bezlotoxumab (BEZ) for prevention of Clostridium difficile infection (CDI) recurrence (rCDI): outcomes in patients with substantial renal impairment (SRI). Open Forum Infect Dis. 2017;4(Suppl 1): S387.

30. Tang YM, Stone CD. Clostridium difficile infection in inflammatory bowel disease: challenges in diagnosis and treatment. Clin J Gastroenterol. 2017;10(2):112-123.

31. Rodemann JF, Dubberke ER, Reske KA, Seo DH, Stone CD. Incidence of Clostridium difficile infection in inflammatory bowel disease. Clin Gastroenterol Hepatol. 2007;5(3):339-344.

32. Bossuyt P, Verhaegen J, van Assche G, Rutgeerts P, Vermeire S. Increasing incidence of Clostridium difficile-associated diarrhea in inflammatory bowel disease. J Crohns Colitis. 2009;3(1):4-7.

33. Kelly CP, Wilcox M, Glerup H, Aboo N, Eves K, Dorr MB. Characteristics and outcomes in patients with $C$. difficile infection (CDI) and inflammatory bowel disease: bezlotoxumab versus placebo. Gastroenterology. 2017;152(5):S340.

34. Wilcox M, Kelly CP, Glerup H. Characteristics and outcomes in patients with $C$. difficile infection (CDI) and inflammatory bowel disease: bezlotoxumab vs placebo. Paper presented at: ECCMID, 27th European Congress of Clinical Microbiology and Infectious Diseases; 2017; Vienna, Austria.

35. Mikamo H, Aoyama N, Sawata M, Fujimoto G, Dorr MB, Yoshinari T. The effect of bezlotoxumab for prevention of recurrent Clostridium difficile infection (CDI) in Japanese patients. $J$ Infect Chemother. 2018;24(2):123-129.

36. Bezlotoxumab. (MK-6072) Versus placebo in children with Clostridium Difficile infection (CDI) (MK-6072-001); 2018. Available from: https:// ClinicalTrials.gov/show/NCT03182907. Accessed November 27, 2018.

37. Connelly TM, Koltun WA, Sangster W, et al. An interleukin-4 polymorphism is associated with susceptibility to Clostridium difficile infection in patients with inflammatory bowel disease: results of a retrospective cohort study. Surgery. 2014;156(4):769-775.

38. Jiang ZD, Garey KW, Price M, et al. Association of interleukin-8 polymorphism and immunoglobulin $\mathrm{G}$ anti-toxin $\mathrm{A}$ in patients with Clostridium difficile-associated diarrhea. Clin Gastroenterol Hepatol. 2007;5(8):964-968. 
39. Ananthakrishnan AN, Oxford EC, Nguyen DD, Sauk J, Yajnik V, Xavier RJ. Genetic risk factors for Clostridium difficile infection in ulcerative colitis. Aliment Pharmacol Ther. 2013;38(5):522-530.

40. Garey KW, Jiang ZD, Ghantoji S, Tam VH, Arora V, Dupont HL. A common polymorphism in the interleukin- 8 gene promoter is associated with an increased risk for recurrent Clostridium difficile infection. Clin Infect Dis. 2010;51(12):1406-1410.

41. Shaw P, Shen J, Dorr MB, et al. Genome wide analysis reveals host genetic variants that associate with reduction in Clostridium difficile infection recurrence (rCDI) in patients treated with Bezlotoxumab. Open Forum Infect Dis. 2017;4(Suppl 1):S380.

42. Ghantoji SS, Sail K, Lairson DR, Dupont HL, Garey KW. Economic healthcare costs of Clostridium difficile infection: a systematic review. J Hosp Infect. 2010;74(4):309-318.

43. Lucado J, Gould C, Elixhauser A. Clostridium difficile infections (CDI) in hospital stays, 2009: statistical brief \#124. Healthcare Cost and Utilization Project (HCUP) Statistical Briefs. Rockville, MD: Agency for Healthcare Research and Quality (US); 2006.

44. Prabhu VS, Dubberke ER, Dorr MB, et al. Cost-effectiveness of bezlotoxumab compared with placebo for the prevention of recurrent Clostridium difficile infection. Clin Infect Dis. 2018;66(3):355-362.
45. Lam SW, Neuner EA, Fraser TG, Delgado D, Chalfin DB. Cost-effectiveness of three different strategies for the treatment of first recurrent Clostridium difficile infection diagnosed in a community setting. Infect Control Hosp Epidemiol. 2018;39(8):924-930.

46. Deshpande A, Pasupuleti V, Thota P, et al. Risk factors for recurrent Clostridium difficile infection: a systematic review and meta-analysis. Infect Control Hosp Epidemiol. 2015;36(4):452-460.

47. Abou Chakra CN, Pepin J, Sirard S, Valiquette L. Risk factors for recurrence, complications and mortality in Clostridium difficile infection: a systematic review. PLoS One. 2014;9(6):e98400.

48. Garey KW, Sethi S, Yadav Y, Dupont HL. Meta-analysis to assess risk factors for recurrent Clostridium difficile infection. J Hosp Infect. 2008;70(4):298-304.

49. D'Agostino RB, Collins SH, Pencina KM, Kean Y, Gorbach S. Risk estimation for recurrent Clostridium difficile infection based on clinical factors. Clin Infect Dis. 2014;58(10):1386-1393.

50. Lexicomp. Bezlotoxumab: Drug Information. Waltham, MA: UpToDate; 2018.
Infection and Drug Resistance

\section{Publish your work in this journal}

Infection and Drug Resistance is an international, peer-reviewed openaccess journal that focuses on the optimal treatment of infection (bacterial, fungal and viral) and the development and institution of preventive strategies to minimize the development and spread of resistance. The journal is specifically concerned with the epidemiology of antibiotic

\section{Dovepress}

resistance and the mechanisms of resistance development and diffusion in both hospitals and the community. The manuscript management system is completely online and includes a very quick and fair peerreview system, which is all easy to use. Visit http://www.dovepress.com/ testimonials.php to read real quotes from published authors. 